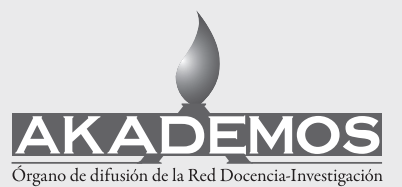

ISSN: $1995-4743$

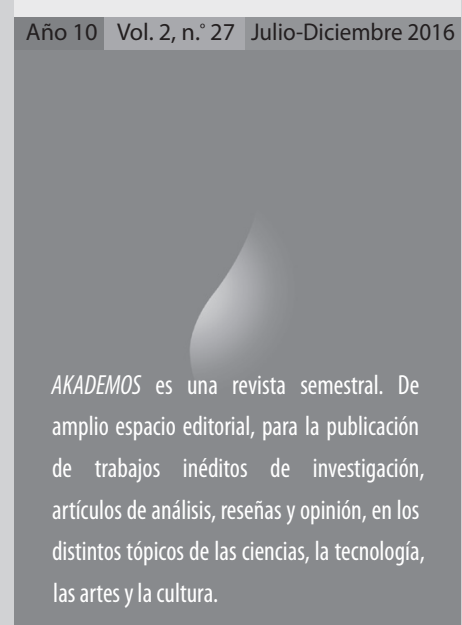

San Salvador, El Salvador, Centroamérica

\title{
Aspectos generales a considerar en la evaluación de proyectos
}

Francisco Sorto Rivas

Master en Economía, Docente Investigador de Facultad de

Posgrados y Educación Continua

fran.sorto@gmail.com

\section{Resumen}

Este breve trabajo está centrado en la identificación de costos que deben incluirse como parte del flujo de caja utilizado para evaluar proyectos de inversión privada $y$ la importancia que tiene la capitalización de los costos cuando la parte preoperativa tiende a extenderse por más de un año; aunque para entender el valor de la selección de activos productivos, para las empresas, me pareció conveniente darle un poco de contexto gerencial.
Palabras clave: Costos Hundidos, Costos Incrementales, Costos de Oportunidad, Costos Relevantes, Valor Cronológico del Dinero.

\section{Introducción}

La realización de un proyecto de inversión responde, normalmente, a la identificación de un problema u oportunidad que deman$\mathrm{da}$ actividades adicionales a las tareas normales en la empresa, ya que, evidentemente, éstas han resultado infructuosas para solventarlo, en el primer de los casos, o aprovechar su potencial, en el caso del segundo. 
Dependiendo de la seriedad con que se haya hecho el ejercicio de formulación y la identificación de los alcances del proyecto, más confiables serán los resultados de la evaluación.

Si no se hubiera profundizado suficientemente en costear las actividades de la Estructura de Descomposición del Trabajo (EDT) o si los hitos definidos en ella no correspondieran razonablemente con la realidad, los presupuestos elaborados para medir la inversión inicial de un proyecto pueden alejarse del dato futuro, por ejemplo.

Es por esa razón que es recomendable, antes de iniciar la labor de evaluación, verificar la calidad de la información utilizada y la robustez de las proyecciones contenidas en la formulación del proyecto, la confiabilidad de los estudios realizados en su formulación definitiva y la presupuestación, en general, comenzando por la validación de las estimaciones de la demanda y localización del proyecto.

Esto es así porque el sobredimensionamiento de la escala de producción, un diseño inadecuado de la distribución en planta o una localización inconveniente de la misma pueden impedir la recuperación de la inversión realizada.

\section{Planeación estratégica, formulación y ejecución de proyectos}

Ahora bien, cuando los problemas y oportunidades identificados por la empresa son determinantes para su viabilidad económica futura, deberían demandar la debida atención de sus autoridades -Gobierno Corporativo-, por constituir factores claves del entorno para la formulación de sus planes estratégicos. Es en este tipo de espacios de discusión y análisis donde se exploran diferentes alternativas técnicas y económicas para corregirlos o aprovecharlos, según sea el caso.

Este tipo de aspectos ambientales, tanto internos como externos, se identifican mediante el acrónimo FODA (Fortalezas, Oportunidades, Debilidades y Amenazas).

Para efectos de determinar cuáles de ellas deberían incluirse en el plan estratégico y cuáles, en otro de contingencia, se apela a la probabilidad de ocurrencia de los eventos identificados como riesgosos, así como el impacto que tendrían sobre la materialización de la visión estratégica de la firma.

A manera de ejemplo, en la siguiente ilustración aparecen clasificadas -hipotéticamente- amenazas potenciales que pudieran afectar el desempeño de una empresa. 


\section{Gráfico 1. Matriz probabilidad impacto}

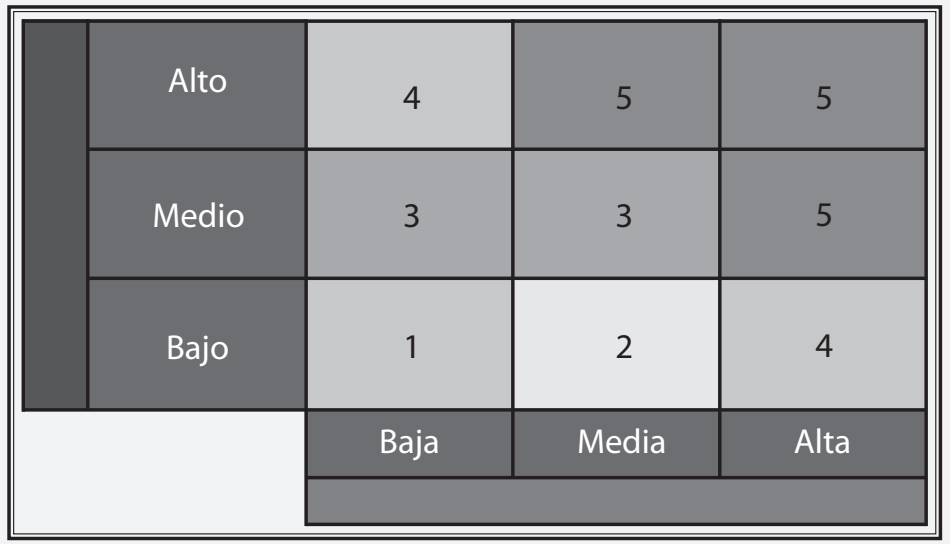

Fuente: Osvaldo Martínez Gómez, Universidad para la Cooperación Internacional, Costa Rica.

Cuando el impacto de los eventos que pudieran suscitarse, sobre las finanzas de una empresa, se consideran altos, y que la probabilidad de que ocurran, también, la corporación debería incluir acciones estratégicas en sus planes, a efecto de enfrentarlas de forma prioritaria; ese sería el caso de los eventos que aparecen en la esquina superior derecha.

En el caso de la esquina superior izquierda, la empresa debería contar con planes de contingencia.

Dicho ejercicio se realiza para las otras tres categorías incluidas en el ejercicio de definición del Plan Estratégico (Debilidades, Oportunidades y Fortalezas).

Una vez definido el Plan Estratégico, se comienzan a gestar perfiles de proyectos que se convertirán, eventualmente, en estudios de factibilidad para la asignación de los recursos corporativos (presupuesto de capital).

En tal sentido, los proyectos deben interpretarse como el medio natural para implementar la visión estratégica de las entidades, a efecto de cambiar su situación actual, por otra considerada deseada.

En los planes estratégicos se identifican, grosso modo, las ideas principales que se traducirán en proyectos y que se implementarán, gradualmente, mediante planes operativos anuales (POA). Ellos contienen, a su vez, programas y proyectos.

Claro está que los planes estratégicos deben guardar estrecha relación con la misión de las organizaciones. Esto es particularmente 
cierto para las entidades públicas, las cual han sido creadas para implementar políticas públicas específicas.

No obstante lo anterior, no resulta extraño observar que determinadas entidades implementen proyectos que no encajan explí- citamente en sus respectivas misiones, pero que pueden resultar complementarias a ellas.

Hay que tener presente, entonces, que existe cierto despliegue de los planes estratégicos hacia la ejecución de proyectos, como la unidad más básica para su concreción.

\section{Gráfico 2. Despliegue de los planes estratégicos}

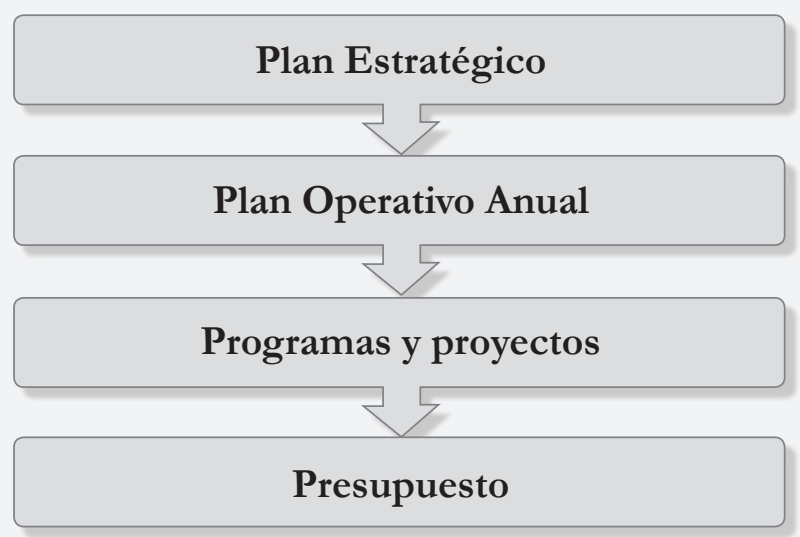

Fuente: Elaboración propia.

Naturalmente que dicho despliegue hacía la formulación, evaluación y su eventual ejecución se le deben asignar recursos apropiados (presupuestos); caso contrario, no sería realista el Plan. Al elaborar los presupuestos de los proyectos a desarrollar en cada ejercicio fiscal futuro se va conformando el presupuesto de capital de la empresa; de no contarse con recursos propios suficientes deberán explorarse fuentes de financiamiento, incluyendo nuevos llamamientos de capital.
A continuación se presenta un esquema útil para entender la estrecha relación que hay entre el presupuesto y los Planes Estratégicos de una empresa, al pasar de éstos a metas de desempeño cuantificables y su seguimiento mediante indicadores.

Es aquí donde el uso del Cuadro de Mando Integral desempeña un papel clave para evitar desviaciones significas respecto al Plan Operativo Anual, como aparece a continuación. 
Gráfico 3. Plan estratégico y presupuesto

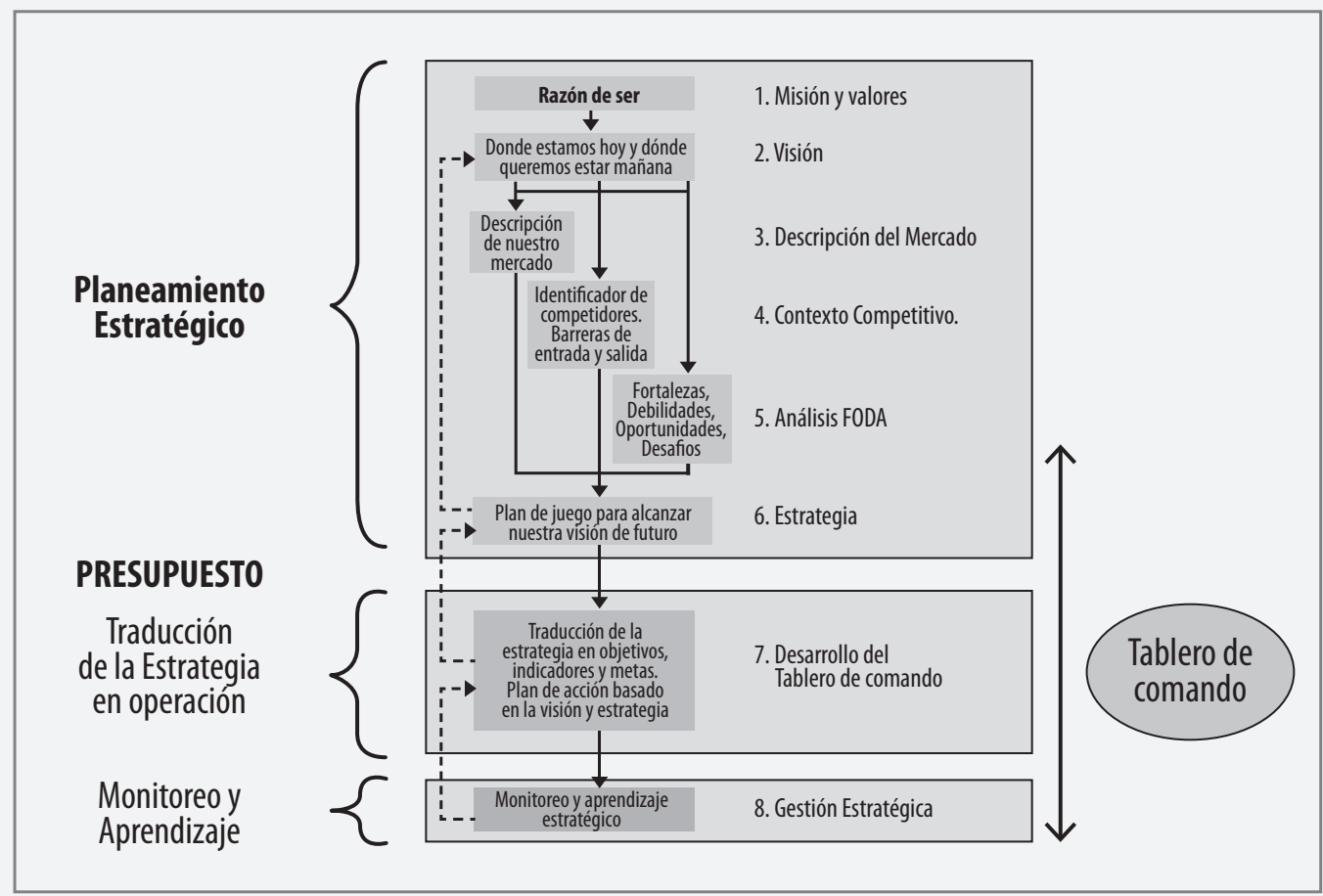

Fuente: F. Casares.

La gestión estratégica supone, entonces, la mejora continua (aprendizaje) como parte de la asignación intertemporal de recursos, la ejecución presupuestaria anual y el desarrollo de los proyectos. Sobre esta relación entre los Planes Estratégicos y su gestión se puede ahondar mucho, pero nos desviaríamos del tema de interés principal de este trabajo, que corresponde a la delimitación apropiada de las transacciones registradas en el flujo de caja y el reconocimiento del costo de oportunidad en el ejercicio.
En tal sentido, al retomar el tema puntual de evaluación de proyectos, podemos decir que muchas veces, su formulación puede demandar algún tiempo para pasar de ideas a estudios de factibilidad, los cuales se nutren de estudios de mercado, técnicos, ambientales, jurídicos, administrativos, fiscales y económicos, entre los más significativos.

Una vez tomada la decisión de invertir en un proyecto inician las erogaciones importantes, con la expectativa de recuperarlas, 
con creces, mediante la comercialización de los bienes o servicios elaborados por el proyecto, como negocio en marcha.
Esto significa que, por practicidad, los proyectos se pueden dividir en etapas o fases, tal como se presenta a continuación:

\section{Gráfico 4. Fases de un proyecto}

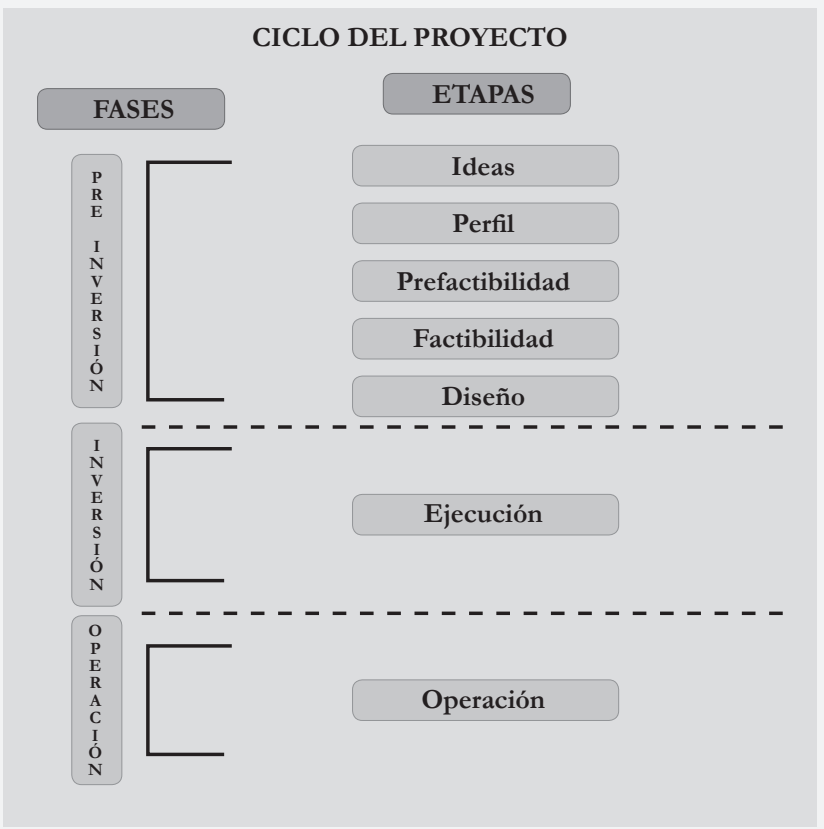

Fuente: CEPAL.

En la medida que avanza el desarrollo de una idea y se convierte en perfil, las entidades ejecutoras destinan más recursos a depurar y precisar las estimaciones de cada componente (diseño, producción, comercialización, etc.) del proyecto; hasta alcanzar su diseño final.

Pero, aun habiendo destinado una cantidad importante de recursos a la elaboración de estudios de prefactibilidad, los inversionistas pueden decidir no ejecutar un proyecto, de tal manera que los gastos incurridos para su formulación y evaluación resultan irrelevantes al decidir si se realiza o no la inversión, por lo tanto, los costos incurridos en estudios no se incluyen en el flujo de caja del proyecto.

\section{Observaciones generales sobre los costos de un proyecto}

Para evaluar cualquier emprendimiento es necesario identificar, apropiadamente, los 
ingresos y erogaciones incurridas al implementarlo; caso contrario, esta herramienta de gestión financiera perdería su utilidad y no garantizaría una asignación eficiente de los recursos escasos de la entidad.

Naturalmente que para la formulación e implementación de un proyecto habrán erogaciones previas a la entrada en operaciones del proyecto y otras que se realizarán durante la fase de operaciones.

\section{Costos relevantes}

Es importante tener presente que muchos gastos previos no se toman en cuenta para el análisis, por considerarse costos hundidos; aunque resulta frecuente observar que se omiten, equivocadamente, desembolsos previos por estar registrados contablemente como gastos - no inversión-; aquí encontramos, por ejemplo, gastos asociados con la compra de un terreno (inversión), como el pago de impuestos por la transferencia de la propiedad del mismo, tasas municipales y derechos de registros; de igual manera se puede citar el pago de primas de seguro sobre activos adquiridos como inversión: vehículos, bienes inmuebles y activos diversos. Dichos gastos son parte de la inversión inicial.

No obstante lo anterior, hay erogaciones previas que no se incluyen en la evaluación de un proyecto, a pesar de haberse contabilizado como inversiones desde un principio, debido a que pueden carecer de utilidad para éste; en esta categoría se encuentra, por ejemplo, el valor de construcciones existen- tes en un terreno, las cuales tendrán que demolerse para poder utilizarlo en el proyecto.

Esa habilidad de discriminar entre gastos elegibles como parte de la inversión inicial y del flujo libre de efectivo (fase operativa), puede hacer la diferencia entre tomar buenas o malas decisiones de inversión, así como entre crear o destruir el valor de la empresa al ejecutarlos.

\section{Costos hundidos}

En ese mismo sentido, los gastos efectuados en estudios de la factibilidad se consideran Costos Hundidos. "No debería incluirse en los flujos porque cualquiera que sea la decisión que se tome, habrá que pagarlos igual".

El propósito de mencionar dichos ejemplos busca enfatizar en el hecho que separar gastos e inversiones no es una labor mecánica; Sapag Chain, especialista en proyectos y referente para América Latina en esta materia, es claro al señalar que inversiones previas, imposibles de recuperar, no deben influir en la toma de decisiones de inversión y que los estudios de factibilidad son costos hundidos. ${ }^{1}$

\section{Costos incrementales}

Asimismo hay gastos previos que no se verán afectados por el proyecto, teniéndose que omitir del análisis financiero; pero si se vieran afectados, el valor a considerar co-

1 Proyectos de inversión. Formulación y evaluación. Prentice Hall, Chile 2011. 
rrespondería sólo a la diferencia en el gasto total, es decir, se consideran como relevantes sólo los valores incrementales.

\section{Costos proporcionales}

Por otro lado, habrán gastos que tendrían que prorratearse al existir erogaciones globales para varios proyectos, cuando sólo algunos de ellos terminan ejecutándose; este sería el caso de gastos de registros de terrenos más extensos que el destinado finalmente a un proyecto en particular. Lo mismo sucedería con los Impuestos de Transferencia de una propiedad utilizada, parcialmente, para algún emprendimiento.

\section{Costos de oportunidad}

En línea con lo comentado anteriormente, en algunas ocasiones el valor a considerar como parte de un flujo de caja no corresponde, necesariamente, a ingresos o desembolsos en efectivo, sino que al sacrificio efectuado al destinar activos propios al desarrollo de un proyecto; en tales casos no se recurre directamente al valor en libro de los activos asignados al proyecto, sino que al de oportunidad; puede suceder que se disponga de una oferta de compra en firme o se cuente con precios de mercado confiables que sugieran que el valor de algunos activos destinados a un proyecto difieren de su valor contable.

En esos casos, cuando dichos activos se destinan al proyecto se estaría renunciando, implícitamente, a un valor superior al registrado en la contabilidad, por lo tanto, el ingreso potencial sacrificado tendría el carácter de costo de oportunidad y tendría que tomarse en cuenta al determinar su inversión inicial, por ejemplo. Esto ocurre, normalmente, con los terrenos que ganan plusvalía con el tiempo.

De igual manera, si se esperaba recibir un ingreso futuro, pero se compensa contra un gasto que debe hacerse para desarrollar el proyecto, éste debe incluirse como costo del proyecto, aunque no se haya registrado ningún movimiento de efectivo en ese momento.

Se abre así una diferencia entre el análisis contable y el financiero, donde predominan principios de reconocimiento de ingresos y egresos distintos; en el caso de la contabilidad se trabajaba con valores devengados y en evaluación de proyectos, con valores percibidos (efectivo).

Congruente con lo anterior, cabe mencionar que al analizar flujos intertemporales, en finanzas, es necesario considerar el valor cronológico del dinero en la toma de decisiones; en contabilidad no, donde la comparación entre cuentas de balance y de resultado corresponde al mismo ejercicio fiscal (razones financieras).

\section{El valor cronológico en el tiempo en la evaluación de proyectos}

No está de más recordar aquí que, la evaluación de un proyecto consiste en identificar todos aquellos costos, gastos e ingresos resultantes de su implementación; procurando que los ingresos excedan los costos de 
ejecución; representando aquí la diferencia entre ingresos y egresos, la rentabilidad de los inversionistas.

De ahí se deduce el indicador utilizado, por excelencia, para evaluar la factibilidad de un proyecto, el Valor Actual Neto (VAN) o Valor Presente Neto (VPN), el cual consiste en comparar la inversión necesaria para ejecutar un proyecto (I) y el flujo de caja neto esperado de la explotación de los activos productivos adquiridos para su desarrollo.
No obstante lo anterior, como en el análisis se combinan flujos de caja erogados o percibidos en diferentes momentos en el tiempo, es imposible, técnicamente hablando, comparar directamente los referidos valores.

Para hacerlo es necesario recurrir a la teoría del Valor Actual, que toma en cuenta el valor cronológico del dinero y el interés compuesto para capitalizar erogaciones pasadas y para descontar flujos futuros, tal como se muestra en la siguiente gráfica:

\section{Gráfico 5. Metodología de evaluación de proyectos}

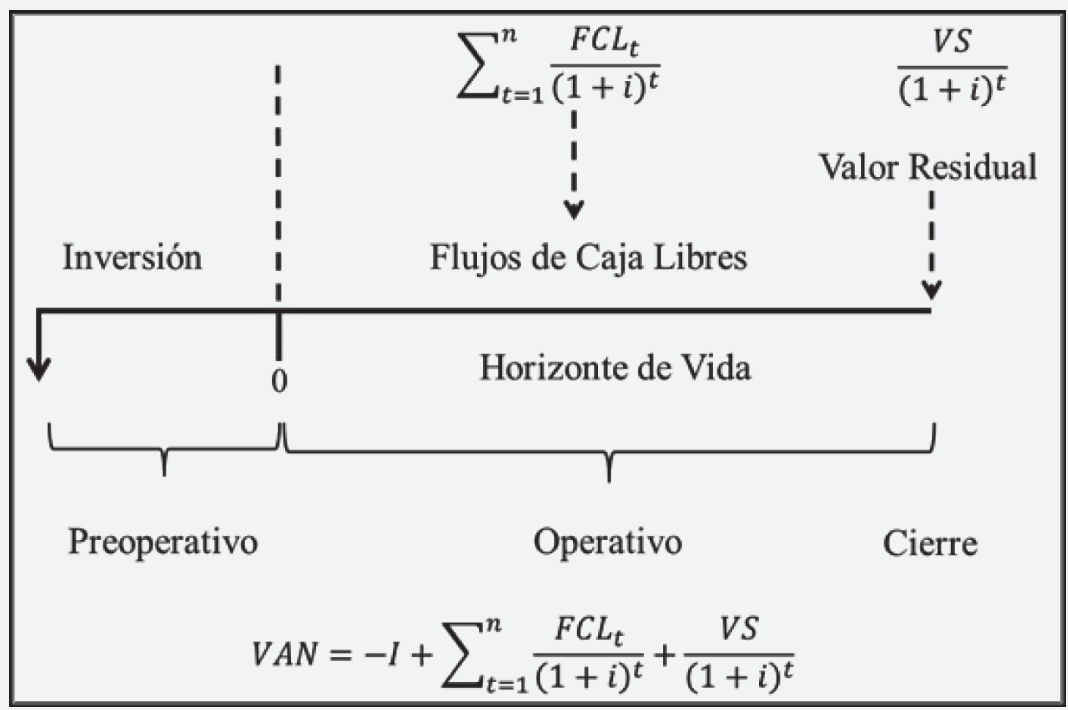

Fuente: Elaboración propia.

Como se puede apreciar en el Gráfico 5 -que corresponde a una línea del tiempo-, el momento presente aparece identificado como año cero (0); los valores a la izquierda, son el pasado; mientras que los de la derecha, el futuro.

A la duración del proyecto, para efecto de valoración, se le denomina Horizonte de Vida 
y permite evaluar los costos y beneficios del proyecto durante un período predefinido, indistintamente que la duración real del negocio supere ese horizonte. Dicha duración tiene, por lo tanto, una finalidad operativa.

Cuando la fase pre operativa es menor a un año, no es necesario capitalizar los desembolsos efectuados antes del inicio de operaciones, pero cuando los proyectos demoran más tiempo -antes de iniciar operaciones-, los costos pasados deben capitalizarse para llevarlos al año cero.

En el caso de los ingresos futuros, éstos deben descontarse para compararlos con la inversión del año cero.

La tasa de interés utilizada para capitalizar y descontar flujos puede ser distinta, dependiendo de las alternativas reales de inversión que se tengan.

$\mathrm{Al}$ descontar los flujos futuros cabe esperar que su importancia relativa vaya disminuyendo en la medida que se alejan del presente, por lo que, la velocidad de recuperación de las inversiones mediante la obtención de ingresos netos incide, significativamente, en los resultados de la evaluación.

Para finalizar hay que enfatizar que el propósito de la Evaluación de Proyectos consiste en apoyar a la dirección de una corporación en la asignación eficiente de los recursos aportados por los accionistas, considerando que las utilidades obtenidas se originan de la explotación de los activos productivos y que, en la medida que se seleccionen aque- llos que prometan corrientes de ingresos marginales superiores a sus costo de adquisición y operación, se estará creando valor de mercado para la empresa.

De ahí la importancia de evaluar la contribución marginal de los activos adquiridos, al valor económico de la empresa o corporación, en el tiempo.

\section{Conclusiones}

Los proyectos representan el medio natural para implementar la visión estratégica de las empresas, las cuales apreciamos en sus $\mathrm{Pla}-$ nes Estratégicos y Planes Operativos Anuales.

La evaluación de los proyectos representa, además, un ejercicio fundamental para la asignación eficiente de los recursos corporativos; contribuyen a la toma de decisión sobre la adquisición de activos productivos de cuya explotación se espera recuperar, con creces, el valor invertido en ellos.

Es por esa razón que se debe tener claro qué flujos deben incluirse en el análisis y cuáles deberían ser su valor económico, no necesariamente contable.

Además, es importante considerar el valor del dinero en tiempo para efectos de análisis, ya que éste cambia en la medida que nos alejamos del inicio de la operación del proyecto, tanto si nos lo hacemos hacia el pasado o hacia el futuro.

Cuando existen valores erogados en años anteriores al inicio de operación de un pro- 
yecto, siendo éstos relevantes para efectos de análisis, se deben capitalizar hasta el inicio de operaciones para poderlos comparar con el flujo de ingresos netos descontados a percibir en el futuro.
De no tomarse en cuanta estos aspectos, poca utilidad tendrían los resultados obtenidos de un ejercicio mal efectuado.

\section{Referencias bibliográficas}

1 Berk Jonathan, DeMarzo Peter, Finanzas Corporativas, Pearson Educación, 2008.

2 García Montoya, Darío; Ingeniería Económica práctica, primera edición; año: 2011; ECOE Ediciones, Colombia.

3 Gutiérrez Carmona, Jairo; Modelos Financieros con Excel, herramientas para mejorar la toma de decisiones empresariales, segunda edición; año 2014; ECOE Ediciones, Colombia.
4 Project Managment Institute; Guía de los fundamentos para la dirección de proyectos (GUÍA DEL PMBOK), Quinta Edición.

5 Nassir Sapag Chain; Proyectos de Inversión: Formulación y evaluación, segunda edición; año: 2011; editorial: Pearson Educación, Chile.

6 Sorto Rivas, Francisco; Estrategias Financieras Corporativas de Largo Plazo; políticas de crecimiento, ISEADE-FEPADE, Primera Edición 2014. 